\section{Neue BMG-Website}

\section{Ministerin im Dialog mit Comicfigur - ein Zukunftsmodell?}

Noch ist es auch unter bmgs. bund.de zu erreichen - das neue Bundesgesundheitsministerium. Doch das „S“ ist schon entfernt (und bei Herrn Müntefering gelandet), auch im Domainnamen: www.bmg. bund.de. Das neue Bundesministerium für Arbeit und Soziales wird unter bmas.bund.de zu erreichen sein. Außer heftigem technischen Umbau der Website ist derzeit nur wenig über relevante Änderungen zu berichten. Aber: Kurz vor Ende der letzten Legislaturperiode hat Gesundheitsministerin Schmidt noch rasch eine Informations-(„Promenbereichen gehören:
- Fragen und Antworten zur elektronischen Gesundheitskarte

- Grundinformationen: Von Sozialgesetzgebung bis Datenschutz - der rechtliche Rahmen der elektronischen Gesundheitskarte

paganda-,)Website zum MautNachfolger „Gesundheitskarte“ online stellen lassen (www.diegesundheitskarte.de). $\mathrm{Zu}$ den The-

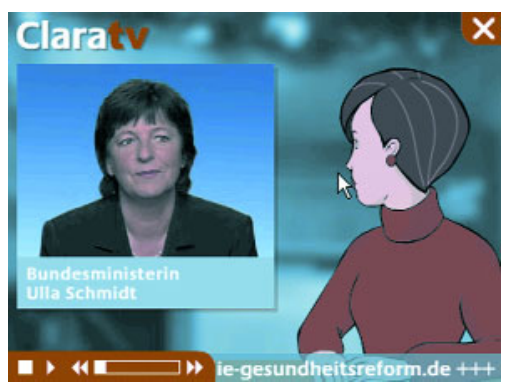

- Bundesgesundheitsministerin Ulla Schmidt zur elektronischen Gesundheitskarte

- Grundfunktionen: Was kann die elektronische Gesundheitskarte?

In der Sektion „Clara TV“ entgleitet die Ministerin dann im Dialog mit einer ministeriumseigenen Comicfigur („Clara“) in virtuelle Lächerlichkeit (www.die-gesundheitskarte.de/clara_tv/video_02/ dsl/index.html).

Rainer H. Bubenzer, Hamburg 